\title{
Measurements of motionally induced voltage in the coastal zone of the Throat of the White Sea
}

\author{
N. A. Palshin ${ }^{1}$, L. L. Vanyan ${ }^{1 \dagger}$, A. M. Poray-Koshits ${ }^{1}$, V. A. Matyushenko ${ }^{2}$, P. Kaikkonen $^{3}$, and J. Tiikkainen $^{3}$ \\ ${ }^{1}$ Shirshov Institute of Oceanology, Russian Academy of Sciences, 117851 Moscow, Russia \\ ${ }^{2}$ Institute of Ecological Problems of the North, Russian Academy of Sciences, 640003 Arkhangelsk, Russia \\ ${ }^{3}$ University of Oulu, Oulu, FIN-90570, Finland
}

(Received December 11, 2000; Revised November 15, 2001; Accepted December 4, 2001)

\begin{abstract}
The theoretical relationship between non-local motionally induced voltages (MIV) and tidal currents is validated with observations of natural low-frequency electric field at the coast of the Throat of the White Sea (northwestern Russia). The Throat of the White Sea is a strait of $50-\mathrm{km}$ width and about $500-\mathrm{km}$ length with depths varying from 20 to $50 \mathrm{~m}$ connecting semi-closed White Sea basin with the Barents Sea. Strong tidal currents providing a reference signal for calibrating coastal measurements of non-local MIV characterize the Throat. The measurements were carried out simultaneously by means of two horizontal receiving on-land and land-sea antennas. Tidally driven MIV dominates in all time series obtained in the coastal zone of the Throat of the White Sea. Monitoring of non-local MIV within the coastal zone could be used for studies of wind tides, residual tidal circulation and temporal variability of a quasi-stationary current. MIV measurements offer an important advantage over traditional oceanographic methods (currents meters, etc.), because it works also in winter period (about 6 months) when the White Sea is covered by ice. The main disadvantage of this technique is a necessity to calibrate non-local MIV with some other oceanographic direct or remote measurements.
\end{abstract}

\section{Introduction}

Natural low-frequency EM fields (with periods longer than an hour) in seas and oceans are induced by sources of two main types: external ionosphere-magnetosphere current systems and internal - the dynamo effect between moving oceanic water and geomagnetic field. Both electric and magnetic field spectra are dominated by tidal and solar daily harmonics, while fields of the internal origin dominate electric field continuum spectra at periods longer than a few days. The motionally induced voltage gives a spatially smoothed measure of water velocity. For a given point it is proportional to the vertically-integrated, seawater conductivity weighted water velocity averaged over a horizontal radius of a few water depths (e.g. Chave and Luther, 1990).

MIV were widely taken up in oceanographic studies during last decade (e.g. Flosadottir and Taira, 1997; Vanyan et al., 1998). The most effective tools for long-term measurements of an electric field in ocean and seas are cross-stream submarine cables, for such cables, the quantity is also integrated along its length and the measured voltage is approximately proportional to integral water transport through the strait. MIV measurements enable studies of temporal variability of water transport through the straits (e.g. Larsen, 1992). Practically, the number of submarine cables available for the scientific use is limited and their location is not optimal for oceanographic application.

Traditional application of MIV field envolves measure-

Copy right (C) The Society of Geomagnetism and Earth, Planetary and Space Sciences (SGEPSS); The Seismological Society of Japan; The Volcanological Society of Japan; The Geodetic Society of Japan; The Japanese Society for Planetary Sciences. ments of local electric field (e.g. Chave and Luther, 1990). In the presence of lateral heterogeneities of water velocity or/and near-surface conductivity a non-local electric field is also generated (Voit et al., 1987; Palshin et al., 1996). The non-local electric field could be significant and in some cases (e.g. coast effect) it could be even greater than the local one (Vanyan et al., 1992). Intense anomalies of MIV are caused by the electric charges generated by lateral inhomogeneities of sea currents and/or electrical conductivity associated with coastline. Therefore, MIV could be monitored by an optimally located and oriented on-land horizontal antenna (Harvey et al., 1977, Palshin et al., 1997). The existence of $\mathrm{M}_{2}$ tidal constituent was separated also from the telluric field measured far in-land in northern Germany (Junge, 1988). Thus, there is theoretical and experimental evidence that MIV could be measured within coastal anomaly, where the contribution of MIV is expected to be large enough to be detected.

The Throat of the White Sea connecting the semi-closed White Sea basin with the Actric ocean (Barents Sea) was chosen for evaluation of the non-local MIV within the coastal zone (see Fig. 1). The Throat of the White Sea is a strait of $50-\mathrm{km}$ width and about $500-\mathrm{km}$ length with depths varying from 20 to $50 \mathrm{~m}$. The mean salinity is about 25 PSU during the whole year and the water temperature is about $7-9^{\circ} \mathrm{C}$ during summer. Strong tidal currents with well-pronounced semi-lunar harmonic $\mathrm{M}_{2}$ characterize the Throat of the White Sea. Spatial distribution of maximal tidal current velocities is shown at Fig. 2. Tidal

\footnotetext{
${ }^{\dagger}$ Prof. Leonid L. Vanyan passed away suddenly on 31 October 2001.
} 


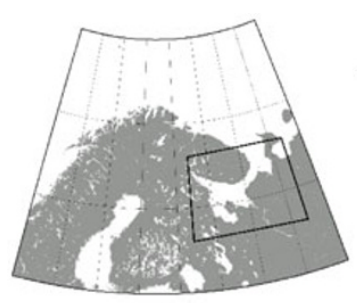

The Barents Sea

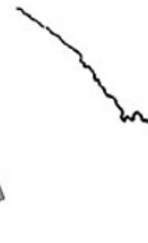

Kola Peninsula

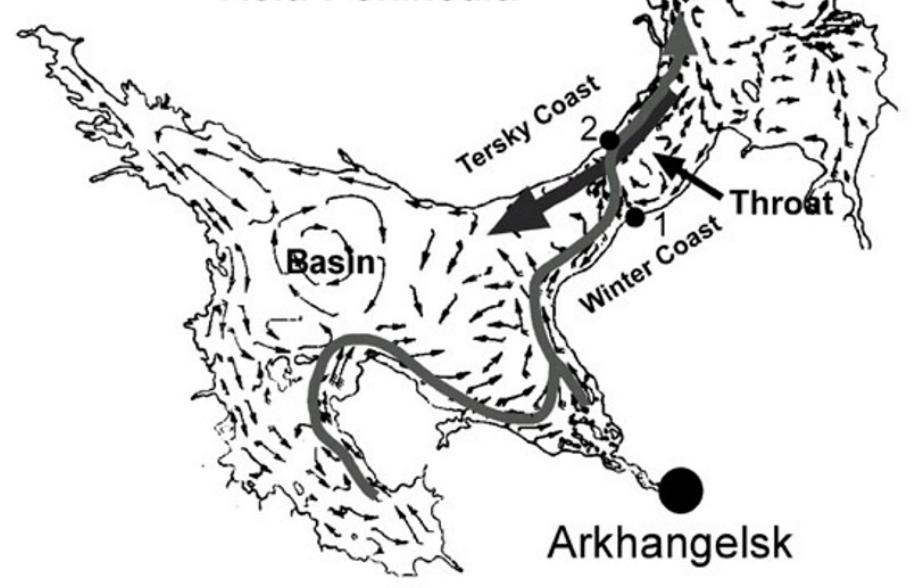

$0 \quad 50100 \mathrm{~km}$

Fig. 1. The scheme of main quasi-stationary currents in the White Sea and the location of observation points. The warm White Sea Current is shown in gray and cold compensatory Barents Sea Current is shown in black. 1: Intsy Lighthouse, 2: Sosnovets Island (Annotated Atlas, 1991).

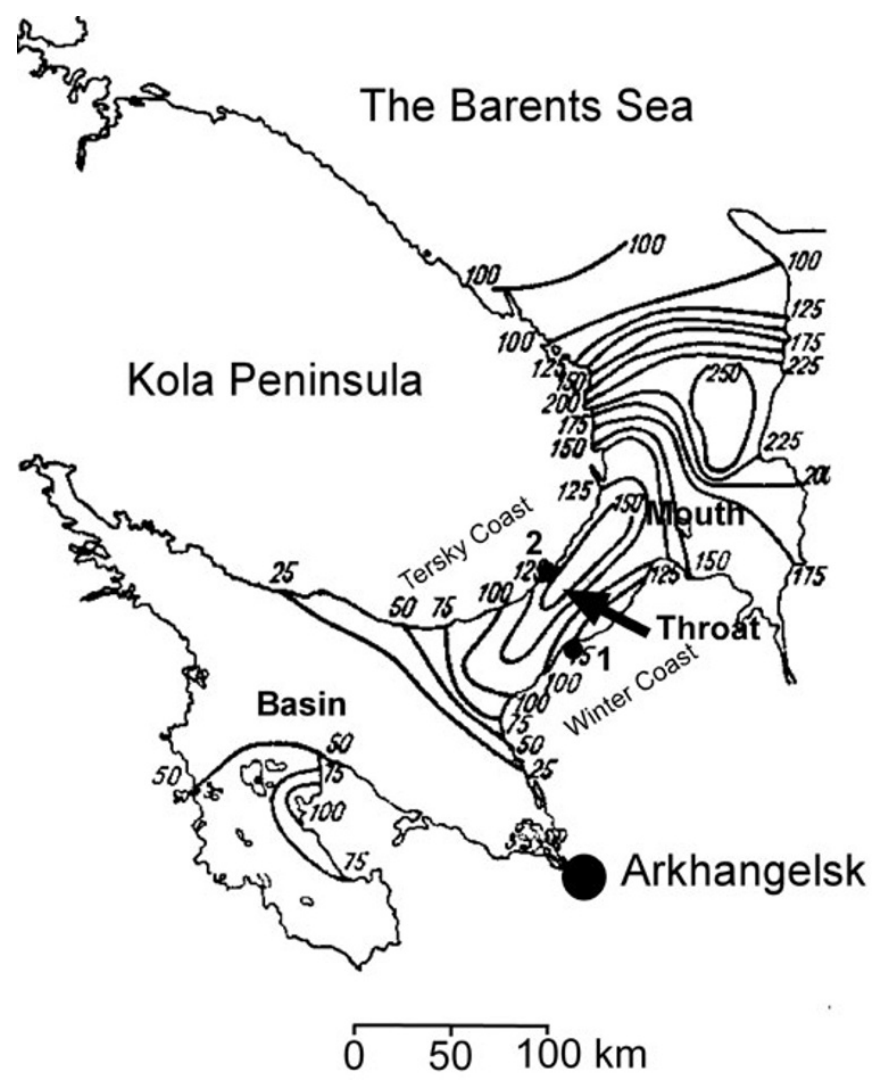

Fig. 2. Maximal velocities of tidal currents in the White Sea in cm/s. 1: Intsy Lighthouse, 2: Sosnovets Island (Annotated Atlas, 1991). 
currents in the Throat of the White Sea are caused by the tidal wave coming from the Barents Sea. The tidal current velocity reaches $0.75-1.0 \mathrm{~m} / \mathrm{s}$ near the Winter Coast and even greater values near the Tersky Coast. Annotated Atlas (1991) contains all necessary hydrological and hydrophysical information including tables of tidal current velocities and phases for several hydrological stations including the Intsy Lighthouse, where the test measurements were carried out. Thus, the strong, regular and well-studied tidal current in the Throat of the White Sea gives a possibility to scale MIV measurements even without simultaneous direct sea current measurements.

Additionally, strong irregular wind-driven currents are typical in the period from late summer to autumn when northern winds with a speed of up to $15-20 \mathrm{~m} / \mathrm{s}$ are quite usual. Wind-driven currents are comparable with the tidal ones. Significant variation of water level is also associated with this wind-driven current (wind tides).

Two main quasi-stationary currents stand out in the Throat of the White Sea. The first one is the warm surface White Sea Current (WSC) and the second one is the cold deep Barents Sea Current (BSC). The general scheme of near-surface water circulation and pathways of the main of quasi-stationary currents is shown at Fig. 1. WSC flows along the Winter Coast, turns to the north and then flows along the Tersky Coast. Water velocity in WSC reaches 0.3 $\mathrm{m} / \mathrm{s}$, but mainly ranges from 0.1 to $0.15 \mathrm{~m} / \mathrm{s}$. BSC flows along the deepest northwestern part of the Throat along the Tersky Coast.

The quasi-stationary currents are characterized by velocities, which comprise about $10 \%-15 \%$ of tidal current velocities. Water transport through the Throat is determined by river run-off and has a pronounced seasonal maximum in May-June and a minimum in January-February. The total amount of river run-off is about $180-220 \mathrm{~km}^{3}$ per year. The river Severnaya Dvina contributes more than half of the amount. Warm and low-salinity waters flow off the White Sea carrying suspended material and different pollutants, while the cold and high-salinity waters from the Barents Sea flow into the White Sea, compensating the river runoff. Thus, the water exchange controls the most of hydrochemical, hydro-physical and biological properties of the White Sea waters.

The measurements of non-local field have some disadvantages as compared to the traditional technique of measuring the local electric field. The main problem is that the nonlocal electric field being proportional to the local one is "unscaled" and the scale factor cannot be obtained theoretically. It's apparent that due to essential spatial heterogeneity of water velocity and near-surface conductivity the intensity of non-local field also varies spatially. The main objective of these experiments was to check the possibility of measuring and scaling of non-local MIV within coastal zone.

\section{Theoretical Background}

The required background for this paper is basically developed by Sanford (1971), following the work of LonguetHiggins et al. (1954). The important elements are noted here. Taking into account electric current $\mathbf{J}$ continuity:

$$
\operatorname{div} \mathbf{J}=0
$$

and that in general case electric current density $\mathbf{J}$ is given by:

$$
\mathbf{J}=\sigma(\mathbf{E}+\mathbf{v} \times \mathbf{B})
$$

where $\mathbf{v}$ is water velocity, $\mathbf{B}$ is Earth's magnetic field and $\sigma$ is electrical conductivity. Electric field $\mathbf{E}$ in Eq. (2) could be called non-local (anomalous) one, while the expression in brackets comes for the total electric field.

Introducing for quasi-stationary assumption $\operatorname{curl} \mathbf{E}=0$ scalar electric potential $U$ as $\mathbf{E}=-\operatorname{grad} U$, which makes the divergence of the Eq. (2):

$$
\operatorname{div}[\sigma(\mathbf{v} \times \mathbf{B}-\operatorname{grad} U)]=0 .
$$

When the water depth, overburden thickness and thicknesses of the upper resistive layers of the crust are thin compared with the horizontal dimensions, it is reasonable to consider a model consisting of two thin sheets. The first one is a conductive sheet with the conductance $S(x, y)$, i.e. the depth-integrated conductivity of sea water, bottom sediments and on-land conductive overburden. The second is a resistive sheet with depth-integrated resistivity $R(x, y)$ that simulates the resistive part of the Earth's crust. These sheets are underlain by a perfect conductor which simulates the conductive deeper Earth's crust (Palshin et al., 1996).

Then, $2 d$ divergence at the upper sheet can be obtained from (3):

$$
\operatorname{div}_{2}(\sigma \operatorname{grad} U)+\partial j_{z} / \partial z=\operatorname{div}_{2}[\sigma(\mathbf{v} \times \mathbf{B})] .
$$

Taking into account that the scalar potential is zero at the bottom of the resistive sheet one can obtain $j_{z}=-U / R$, where $R$ is depth-integrated resistivity or transverse resistance.

Finally the Eq. (4) after integration along vertical axis gives the following:

$$
\operatorname{div}(S \operatorname{grad} U)-U / R=\operatorname{div}\left[S_{m}\left(\overline{\mathbf{v}}^{*} \times \mathbf{B}\right)\right]
$$

where $S$ is the conductance of the upper sheet including sediments and water layer and $S_{m}$ is the conductance of the moving water and water velocity $\mathbf{v}$ becomes depth-averaged conductivity weighted horizontal water velocity $\overline{\mathbf{v}}^{*}$ :

$$
\overline{\mathbf{v}}^{*}=\frac{\int_{-H}^{0} \sigma \mathbf{v} d z}{\int_{-H}^{0} \sigma d z},
$$

where $H$ is the water depth, $\sigma$ is conductivity of the seawater.

The total horizontal electric field $\mathbf{E}_{t}$ can be calculated as:

$$
\mathbf{E}_{t}=\overline{\mathbf{v}}^{*} \times \mathbf{B}-\operatorname{grad} U
$$

The first term in Eq. (7) is local (or source field) field, while the second one is non-local (or anomalous) field.

Numerical modeling is an essential stage of the study. It makes it possible to estimate both qualitatively and quantitatively the spatial distribution of MIV. The numerical 
modeling technique based on the thin sheet approximation (Vanyan et al., 1992; Yegorov and Palshin, 1994) of the medium was applied to the idealized model of quasistationary currents in the Throat of the White Sea. The model of near-surface conductivity was based on ETOPO5 bathymetry database and existing geological and geophysical data on the sediment cover. Numerical estimations of the electric field in the Throat of the White Sea as well as on it coasts suggest that MIV measurements have considerable promise in this region. The largest on-land non-local MIV values are expected to exist in the central part of the Winter Coast. The calculations were carried out for the depth-averaged water velocity of the main quasi-stationary currents equal to $0.1 \mathrm{~m} / \mathrm{s}$ and the largest estimated on-land MIV value is $0.5 \mathrm{mV} / \mathrm{km}$. The scale of non-local field coastal anomalies are controlled by the adjustment distance $G=\sqrt{S \cdot R}$. Specific values of $S$ and $R$ for the Throat of the White Sea gives $G$ about $30-50 \mathrm{~km}$, while the optimal length of the receiving line is about $5-10 \mathrm{~km}$ (Palshin et al., 1999).

Numerical modeling results indicate that at the coastal zone of the Throat of the White Sea the effect of heterogeneous conductivity dominates and coastal anomalies of the non-local MIV have the same nature as traditional coastal effect; they are caused by electric charges concentrating along the coast (largest conductivity gradient). The largest conductivity gradient zone near The Intsy Lighthouse is located approximately 4-5 km off-shore. Thus, the measured electric field is equal to non-local one and it should have the same sign both on land and in the coastal zone of the Throat.

In spite of the significance of obtained numerical estimations, these results should not be overestimated, since only the experimental field measurements of non-local MIV at the coasts of the Throat of the White could allow getting proper estimation of resolving power of coastal monitoring of MIV in this region.

\section{Test Measurements at the Winter Coast of the Throat of the White Sea}

Test measurements of MIV at the Winter Coast of the Throat of the White Sea were carried out in 1998 and 1999. MIV measurements are based on accurate recording of electric potential difference through a pair of grounded non-polarized electrodes. Low-noise non-polarizable electrodes designed and manufactured in the Institute of Geoelectromagnetic Researches of the Russian Academy of Sciences were used for grounding the receiving antennas (Bogorodsky et al., 1998). The electrodes are of $\mathrm{Pb}-\mathrm{PbCl}_{2}$ type, constructed inside a plastic tube $140 \mathrm{~mm}$ in length and $35 \mathrm{~mm}$ in diameter filled with a $\mathrm{PbCl}_{2}$ electrolyte in gel form with a spiral shaped $\mathrm{Pb}$ wire inside. Electrodes manufactured for the seawater environment have a special design.

The electric potential difference was measured with a DATATAKER DT 50 data logger and stored on a memory card. Input impedance of more than 100 mega $\Omega$ was used in the input amplifier to supply allowable low current limit ( $2 \mathrm{nA})$ for the electrodes in use. The resolution of the record is 16 bits per $1 \mu \mathrm{V}$. The signal was sampled 10 times per second and averages over 1-min intervals were stored in data file. The recording instrumentation was lo- cated in the Lighthouse building. The relatively stable temperature of the recording instrument results in low temperature drift as compared with the instrumentation installed outdoors. An alkaline accumulator battery, which could potentially provide more than a month of continuous operation, was used for powering the DATATAKER. A portable computer with RS-232 interface was used for programming of the DATATAKER and initialization of the recording, as well as for uploading the stored data.

The main problem of long-term electric field measurements is not the parameters of acquisition system or capacity of power units, but the safety of the long wires connecting the electrodes, industrial and natural noise of different origin, which contaminates the signal, and temporal stability of grounding impedance.

\subsection{Field measurements}

The observation site nearby the Intsy Lighthouse, which is located in the central part of the Winter Coast (see Fig. 1), was chosen according to the numerical modeling results (Palshin et al., 1999). The Intsy Lighthouse is $30 \mathrm{~km}$ away from the nearest village, thus relatively low noise is ensured. The Lighthouse is equipped with diesel electric power supply, which allows recharging of batteries.

The first receiving dipole antenna was installed in June 1998. The distance between electrodes amounts to $2500 \mathrm{~m}$, whereas the azimuth is $100^{\circ}$ (clockwise from the north). Unfortunately it was impossible to install the receiving line with optimal parameters because of severe environmental factors. According to the numerical modeling results the preferred direction of the receiving antenna is perpendicular to the direction of the Throat which is about $120^{\circ}$; but since the antenna was to be installed in a swampy area, the only possible direction was chosen. The length of the line was limited by $2500 \mathrm{~m}$ for the same reason. The electrodes were placed additionally in plastic buckets and buried at a depth of about $0.5 \mathrm{~m}$ beneath the ground surface. Permafrost layer at depth about $0.5 \mathrm{~m}$ limited the depth at which electrodes were placed. A telephone type wire was used to connect the electrodes to the recording unit. For the sake of safety the wire was buried at a depth of about $20-30 \mathrm{~cm}$ in a sandy dune on which the Lighthouse is situated. Both electrodes were grounded in the soil of the same kind and at the same depth. Test measurements were carried out during one day (two tidal cycles), due to financial and organizational problems. Delivery of the field team to Winter Coast, its disembarkation and returning back in Arkhangelsk was carried out by the crew of r/v "Akvanavt-2".

In August 1999 during the scheduled research cruise to the Throat of the White sea of $r / v$ "Valentin Korzun," (owned by Northern Department of Federal Hydrometerological Service) a land-sea receiving antenna near the Insty Lighthouse was installed in addition to the on-land antenna. The main objectives of measurements in the year 1999 were directed at (1) development and applying installation technique of land-sea receiving antenna and (2) to carrying out simultaneous electric potential difference measurements with the help of two receiving antennas for detailed studies of non-local MIV at the coastal zone.

The most critical point of 1999-year field experiment was installation of a land-sea antenna. Shallow water 1-2 m 
depth and rocky bottom at the Winter Coast prevents vessels from approaching the coastline closer than $500 \mathrm{~m}$ in the area of location of the Intsy Lighthouse. Additionally, strong winds, surges and currents are frequent for the Throat of the White Sea. A high-density geophysical 7-mm-diameter steel-shielded cable with copper conductor (originally designed for logging measurements), which could survive in severe shallow water conditions, was used for installation of a land-sea antenna. The reel with the cable was arranged at the deck of the vessel. Therefore, the most difficult part of installation was to pull the end of the cable from the vessel, which was not equipped with winches and special embankment boats (pontoons) to the coast. Thus, a special technique was developed and tested. At the first stage the end of a plastic halyard (600 m long) connected to the cable onboard was unreeled by the small boat moving to the coast. On board the vessel the end of the halyard was connected with the cable's end; the connection of the cable and halyard was equipped with the buoyancy to prevent a possibility of trapping the cable in the rocky sea-bottom when dragging. At the second stage the cable end was pulled manually by a plastic halyard to the beach and properly fixed there. After that the cable was unreeled and placed on the bottom by the vessel moving slowly off the coast. And at last, the other end of the cable was grounded at the depth $20 \mathrm{~m}$ and at $4500 \mathrm{~m}$ distance from the coastline. The cable was installed in the direction of $330^{\circ}$, which was approximately perpendicular to the coastline. Electrodes similar to those used for the grounding of the land dipole but designed specially for the sea application were used for grounding the land-sea antenna. The sea-grounding electrode was fixed inside a special concrete to avoid electrochemical noise caused by conventional metal anchors. The land end of this antenna was grounded by the same type of electrode in the sandy beach at the depth about $0.5 \mathrm{~m}$ beneath the low tide water level. The general scheme of the receiving antennas location is shown in Fig. 3.

The fieldwork was carried out within a week. It took two days for the preparations and installation of land-sea antenna. Two more days were used for installation of the short on-land lines connecting the antennas to the acquisition sys-

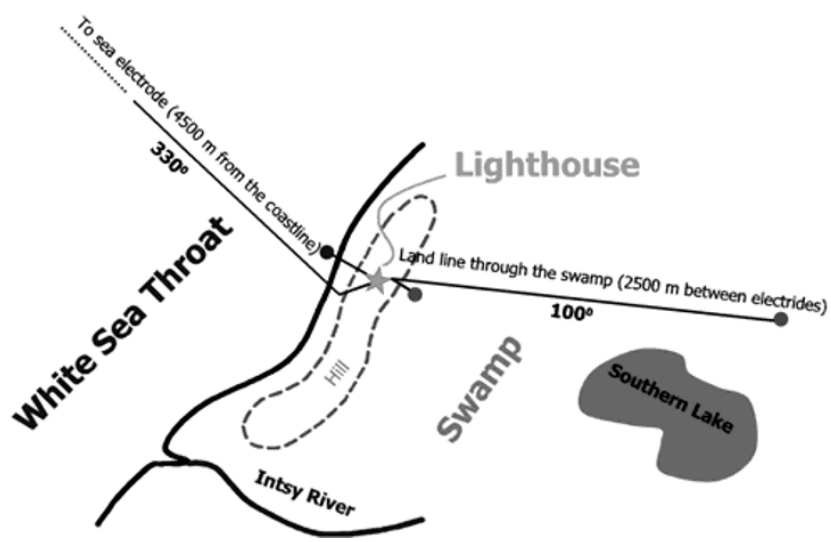

Fig. 3. The layout of the receiving antennas at the Winter Coast of the Throat of the White Sea near Intsy Lighthouse (see Figs. 1 and 2). tem and checking the whole measuring system. Therefore, continuous simultaneous measurements were limited to about three days (6 tidal cycles).

\section{Data Processing and Analysis}

Besides MIV raw electric potential difference measurements contain a geomagnetically induced component and noise. Thus, the main goal of data processing is separation of MIV caused by tidal currents (in our particular case) from the measured raw signal. Since time series obtained are relatively short, a simplified preprocessing procedure, namely a robust estimation of hourly mean values (Hogg, 1979; Larsen et al., 1996), was used. Our previous experience (Palshin et al., 1997) confirmed the efficacy of this technique, which suppresses outliers and non-stationarities in time series. Figure 4 shows an example of the data processing results for the time series obtained by mean of the a landsea receiving antenna. Besides, the robust estimation gives possibility to remove high frequency $\left(<3-4 \cdot 10^{-4} \mathrm{~Hz}\right)$ geomagnetically induced variations (pulsation and partly substorms). Standard deviation values for all time series were also calculated and analyzed. For the further analysis hourly mean time series were used.

At the next stage time series were firstly detrended using second order polynomial function approximation and then were approximated by a set solar and lunar harmonics us-
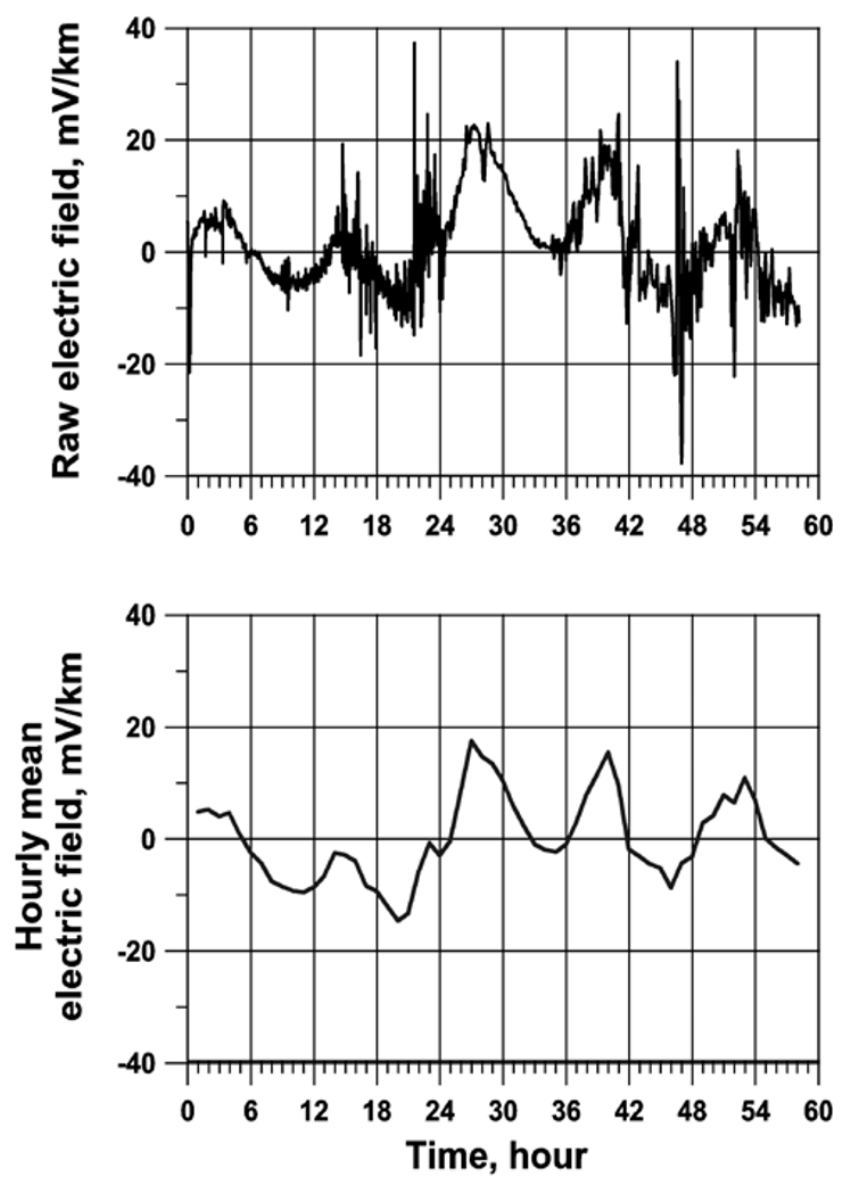

Fig. 4. Raw electric field recorded by the land-sea antenna (upper panel) and hourly means average of the electric field calculated by robust procedure (lower panel). Data collected in August 15-17, 1999. 
ing least square method (LSM). LSM makes it possible to evaluate a statistical significance of different harmonics (e.g. Chave et al., 1989). Secondly, the residual time series were calculated as a difference between detrended hourly mean values and the sum of statistically significant harmonics. The limited duration of measurements and absence of both magnetic field measurements and magnetic observatories in White Sea region did not allow application of more sophisticated procedures for MIV separation (e.g. Larsen et al., 1996). The nearest to Throat of the White Sea magnetic observatory is Oulujärvi IMAGE chain station (International Monitor for Auroral Geomagnetic Effects) (Viljanen and Häkkinen, 1997), which is located about $800 \mathrm{~km}$ to the west at approximately the same magnetic latitude. The magnetic field variations with 1-min sampling measured at Oulujärvi were processed at the same manner as electric potential difference measurements for further joint analysis.

4.1 Geomagnetic variations and high frequency noise

Figure 5 shows a portion of the electric field measured at 1999 by a land receiving antenna and a northern component of the magnetic field at Oulujärvi with 1-min resolution. Long period variations (the sum of diurnal and semidiurnal constituents) were filtered out from the time series. It is clearly seen that time intervals with high frequency noise are correlate with time geomagnetically disturbed time
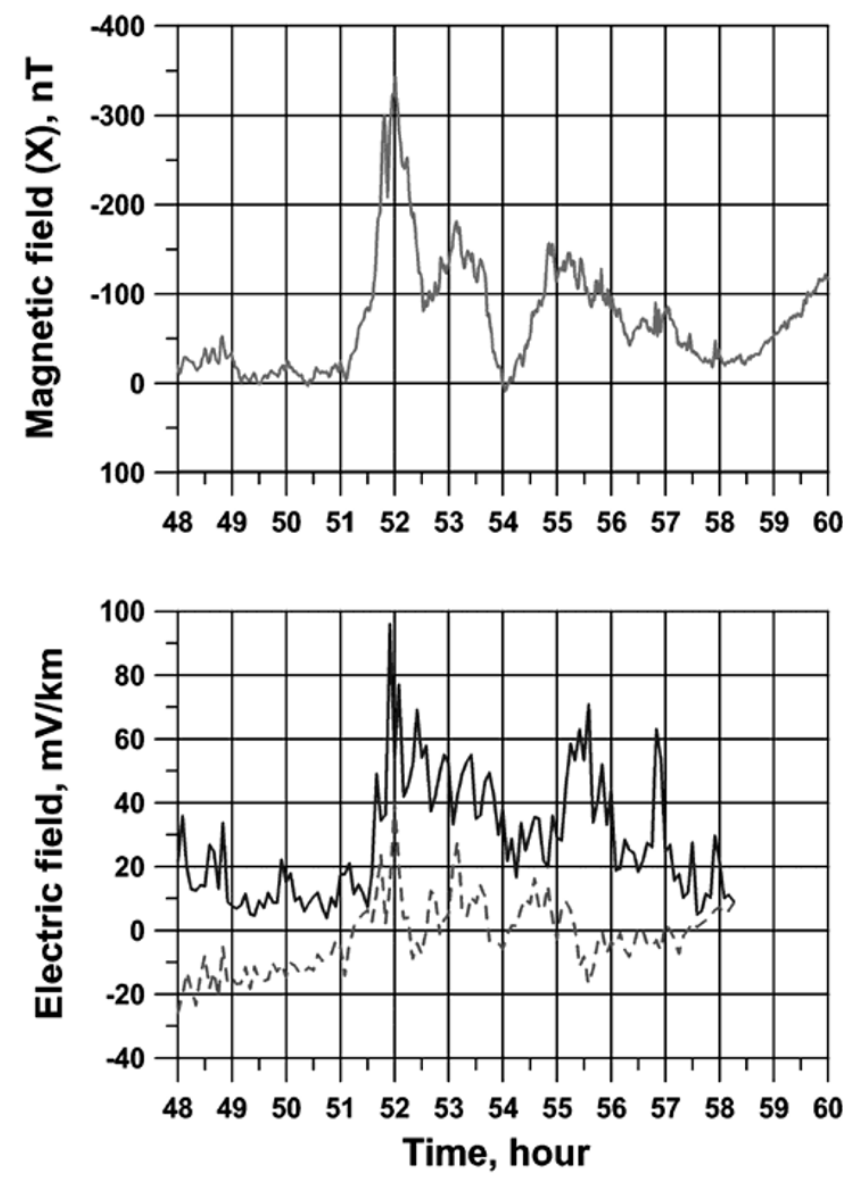

Fig. 5. Part of the raw electric field record obtained by on-land (dashed line) and land-sea (solid line) antennas (lower panel) and the northern component of the magnetic field from Oulujärvi magnetic observatory (upper panel). intervals. In the year 1999 the time of observations coincides with a magnetic storm, which started between 51 and 52 hours of observation. The electric field measured by both antennas and northern component of magnetic field at Oulujärvi are shown at Fig. 5. The magnetic disturbance of northern component of the magnetic field reaches $350 \mathrm{nT}$, while it electric response is about $85 \mathrm{mV} / \mathrm{km}$ (land-sea antenna) and about $35 \mathrm{mV} / \mathrm{km}$ (on-land antenna). The difference between the electric responses measured by sea-land and land antennas may be explained by the difference in antennas orientation, which is about $20^{\circ}$ and by different location and grounding environment (static-shift-type effect). Similar behavior of the variance of magnetic and electric fields within 1 hour intervals also confirms that geomagnetic variations are the main source of high frequency "noise" in electric field measurements for period less than 1 hour. Figure 4 shows an example of robust averaging of the potential difference measurements. Evidently, most of the high frequency noise is removed from time series by means of the rather simple procedure.

\subsection{Lunar and solar constituents and residual field}

Since the main objective of our study is to calibrate onland MIV measurements using the strong and regular tidal
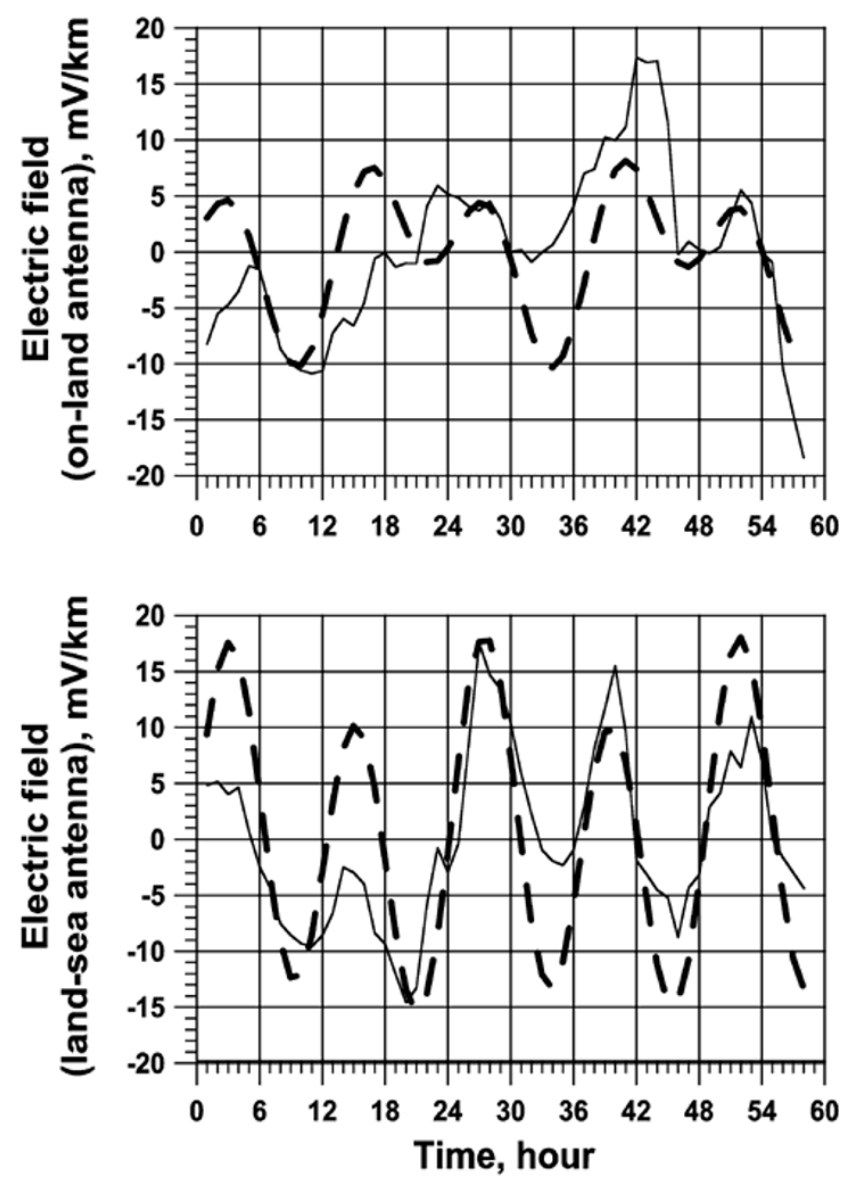

Fig. 6. Results of LSM approximation of averaged electric field collected in August 15-17, 1999. Upper panel shows the electric field recorded by the on-land antenna, while the lower panel shows the electric field recorded by the land-sea antenna. Thin lines are averaged electric field and thick dashed lines are sum of two statistically significant harmonics $\left(\mathrm{S}_{1} / \mathrm{O}_{1}\right.$ and $\left.\mathrm{S}_{2} / \mathrm{M}_{2}\right)$. 


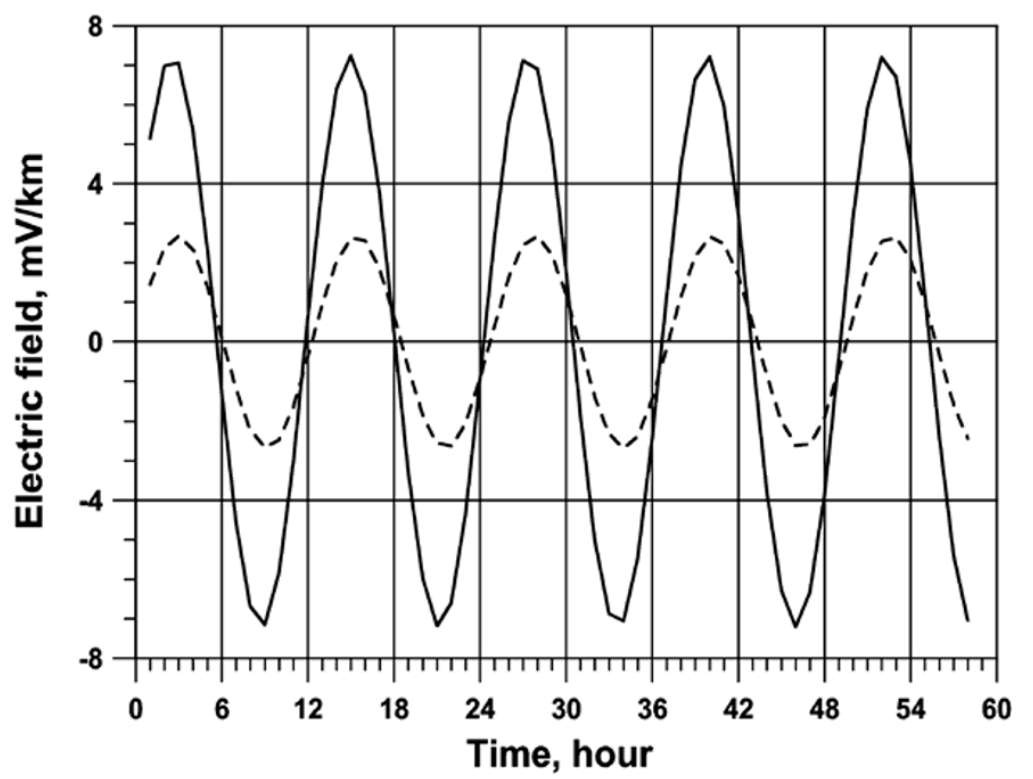

Fig. 7. Comparison of $\mathrm{M}_{2}$ tidal signals separated from electric field recorded by on-land (dashed line) and land-sea (solid line) antennas.

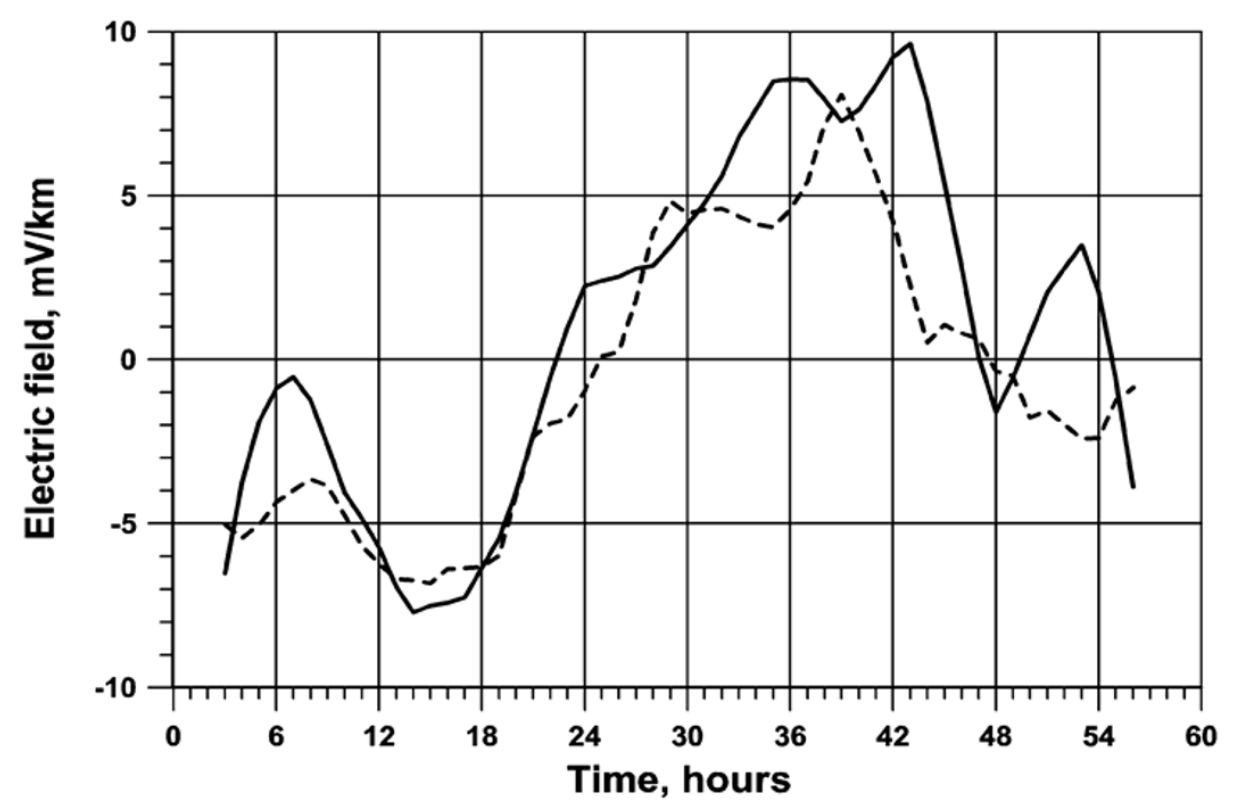

Fig. 8. Comparison of the residual electric field measured by on-land (dashed line) and land-sea (solid line) antennas.

current as a reference signal special attention was paid to solar and lunar constituents analysis. The application of the LSM method confirmed that main semi-diurnal $\mathrm{S}_{2} / \mathrm{M}_{2}$ constituent is statistically significant in all time series obtained. Additionally diurnal variation $\mathrm{S}_{1} / \mathrm{O}_{1}$ is also presented in all time series. Limited duration of the time series gave no possibility to separate $\mathrm{O}_{1}$ and $\mathrm{S}_{1}$ as well as $\mathrm{M}_{2}$ and $\mathrm{S}_{2}$. Tidal parameters tables (phase of $\mathrm{M}_{2}$ tide) from Annotated Atlas (1991) were used for confirmation of the tidal origin of separated $\mathrm{S}_{2} / \mathrm{M}_{2}$ constituent. $\mathrm{S}_{1} / \mathrm{O}_{1}$ constituent was assumed to be mainly of geomagnetic origin, while $\mathrm{O}_{1}$ tidal constituent according to Annotated Atlas (1991) is much smaller than $\mathrm{M}_{2}$. It should be mentioned that a sum of diurnal and semi- diurnal constituents is responsible for the most part of variance of the time series obtained, especially for the data obtained in the year 1998 during the geomagetically quiet time interval and simultaneously during the lunar phase corresponding to high tides (syzygia). Nevertheless we have concentrated our attention on data obtained in the year 1999, because MIV were recorded by means of two antennas.

The results of approximation obtained in the year 1999 are presented at Fig. 7. The amplitude of semi-lunar $\mathrm{M}_{2}$ tidal constituent in MIV measured by means of on-land antenna was about $5.5 \mathrm{mV} / \mathrm{km}$ in June 19,1998 and $2.7 \mathrm{mV} / \mathrm{km}$ in August 15-18, 1999 corresponding to lunar phase. The amplitude of semi-lunar $\mathrm{M}_{2}$ tidal constituent in MIV measured 
by means of the land-sea antenna was estimated to be 7.2 $\mathrm{mV} / \mathrm{km}$. Practically, the land-sea antenna is measuring the sum of the non-local and local field, but in our particular case the latter is smaller due to very shallow water in the coastal zone near the Intsy Lighthouse (Palshin et al., 1996, 1999).

The amplitude of solar daily harmonics was estimated to be of $2.3 \mathrm{mV} / \mathrm{km}$ (19 June, 1998), $4.9 \mathrm{mV} / \mathrm{km}$ (15-17 August, 1999) for the on-land antenna and $4.1 \mathrm{mV} / \mathrm{km}$ (15-17 August, 1999) for the sea-land antenna. This constituent of the electric field is not very important for our studies, because most probably its nature is geomagnetic solar daily variation dominating in magnetic field. The difference in amplitude and phase of the electric field obtained in 1998 and 1999 is explained by different magnetic disturbance level. Some additional signal could be produced by solar tides at the same frequency, but its amplitude is much smaller than the geomagnetically induced part and could not be estimated from short time series obtained.

Additionally a residual field was calculated as a difference between averaged time series and the sum of $S_{1}$ and $M_{2}$ harmonics (see Fig. 8). The residual field resembles the signals in showing similar bahavoir of signals obtained by the on-land antenna and most probably is induced by storm surges.

Thus, the level of useful signal (MIV) is significantly greater than noise level (geomagnetically induced field). The dominance of tidally driven MIV in all time series obtained in the coastal zone of the Throat of the White Sea is the most important result of our test measurements. This fact gives us possibility to calibrate coastal measurements of non-local MIV. As the measurements in the year 1998 were carried out during moon phase corresponding to high tides $(0.75-1.0 \mathrm{~m} / \mathrm{s})$ the scale factor for the on-land antenna is estimated to be on the order of $5-10 \frac{\mathrm{mV} / \mathrm{km}}{\mathrm{m} / \mathrm{s}}$ and as twice as big for the land-sea line according to the results obtained in the year 1999.

When long enough time series are collected more accurate procedures for separation of motionally induced signals can be applied and not only strong tidal and wind-driven currents could be monitored, but quasi-stationary currents as well. The study of wind tides and quasi-stationary currents need also additional efforts for evaluation of the scaling factor, which may differ from one obtained for the tides due to different spatial and temporal scales.

\section{Discussion and Conclusions}

Suitable locations for observation sites can be selected with the help of numerical modeling, which is a useful tool for estimating spatial distribution of local and non-local MIV. On-land MIV observations are based on precise measurements of natural electric field. Hence, the proposed technique is vulnerable to electromagnetic noise, and observation sites should be located far away from industrial areas. The Throat of the White Sea and other coasts of Arctic Seas are poorly populated and, therefore are favorable for application of on-land measurements of non-local MIV.

An installation technique for long $(2-5 \mathrm{~km})$ on-land and sea-land receiving antennas was developed and tested in severe Arctic environment. The land-sea antenna was installed without chartering an expensive specialized cable vessel. The experience obtained during the test measurements is very important for future application of MIV in Arctic Seas and its coasts. The most difficult task during the measurements is to achieve the long-term stability of grounding conditions, which could change dramatically during wintertime, and safety of wires, which are very often damaged by animals, mainly rodents.

Basic requirements are as follows:

(1) shielded cables are to be used to provide the mechanical protection of the antennas and to reduce high frequency noise;

(2) non-polarizable $\mathrm{Pb}-\mathrm{PbCl}_{2}$ or $\mathrm{Ag}-\mathrm{AgCl}_{2}$ electrodes should be used for grounding, and additionally electrodes should be buried below the freezing depth, when grounds are installed in-land, to provide the stability of receiving antennas parameters;

(3) any industrial voltmeter with high input impedance and 24-bit analog-digital converter could be used for data acquisition.

Our experiment showed that a land-sea antenna is preferable, because it is much easier to provide safety of the cable and stability of groundings at the seafloor. But in this case we face another problem - during spring, break up moving ice can rip the cable connecting the on-land based acquisition system and seafloor groundings. To avoid this, and to provide all-year safety of the installation at Arctic Sea coasts, the only possibility is to locate the acquisition system at the seafloor, though it will make the whole system more expensive. Thus, carefully installed and grounded on-land antenna has evident advantages, mostly because it could be installed without renting a vessel and because approximately the same data could be collected.

Data processing procedure based on robust averaging of raw data gave us possibility to obtain reliable results. When long enough time series are collected LSM approximation will give possibility to estimate various tidal constituents and more accurate procedure of separation of motionally induced signals could be applied (Larsen et al., 1996; Fujii and Chave, 1999).

Previous theoretical results showed that a coastline provides an amplification of the MIV component perpendicular to the coastline (Palshin et al., 1996). Electric field measurements with receiving antennas located at both sides of the coastal line give possibility to get experimental estimations of non-local MIV amplified by the coastal effect. The results of the test measurements within coastal zone in the Throat of the White Sea confirmed theoretically predicted MIV level at the coasts of the Throat of the White Sea (Palshin et al., 1999) and showed that a non-local MIV measurements are promising tool for studying temporal variability of sea currents. In particular this technique could be used for studying tidal currents, residual tidal circulation and wind-driven currents. There is also a possibility to get information on quasistatic currents long-term (meso-scale and seasonal) variability.

MIV measurements offer an important advantage over traditional oceanographic methods (current meters, etc.), because it works also in winter period (about 6 months) when the White Sea is covered by ice. That is especially valuable 
for the arctic regions. The main disadvantage of this technique is a necessity to calibrate non-local MIV with some other oceanographic direct or remote measurements. But its flexibility and low cost compensate to certain extent the above mention disadvantage.

Acknowledgments. The study would have been impossible without unconditional help of the personnel of the Intsy Lighthouse, operated by the Russian Navy, crews of $r / v$ "Akvanavt-2" owned by Northern Mining Company and r/v "Valentin Korzun" owned by the Northern Department of the Federal Hydro-Meteorological Service, the Department of Geophysics of Oulu University, which provided an acquisition system, and many other individuals, who backed our field work in every possible way. The Russian Foundation for Basic Researches and the Institute of Ecological Problems of the North (Arkhangelsk) provided financial support during 1996-1999.

\section{References}

Annotated Atlas, Oceanography and biological productivity of the White Sea, Murmansk, 216 pp., 1991 (in Russian).

Bogorodsky, M. M., S. P. Gaidash, Yu. I. Kuksa, and A. M. Poray-Koshits, Electrodes for on-land and seafloor measurements of electric field in Baikal Lake and White Sea, IV International Workshop "Modern technique and tools of oceanographic studies", Moscow, November 24-26, Book of Abstracts, p. 14. 1998.

Chave, A. D. and D. S. Luther, Low-frequency, motionally induced electromagnetic fields in the ocean, 1. Theory, J. Geophys. Res., 95, 7185$7200,1990$.

Chave, A. D., J. H. Filloux, D. S. Luther, L. K. Law, and A. White, Observation of the motional electromagnetic fields during EMSLAB, $J$. Geophys. Res., 94, 14152-14166, 1989.

Flosadottir, A. and K. Taira, Observations of ocean currents using submarine cables, Proceedings of International Workshop on scientific use of submarine cables, Feb. 25-28, Okinawa, Japan, 10-15, 1997.

Fujii, I. and A. D. Chave, Motional induction effect on the planetary scale: geoelectric potentials in the eastern North Pacific, J. Geophys. Res., 104, 1343, 1999.

Harvey, R. R., J. C. Larsen, and R. Montaner, Electric field recording of tidal currents in the Strait of Magellan, J. Geophys. Res., 82, 3472-3476, 1977.

Hogg, R. V., An Introduction to Robust Estimation, in Robustness in Statistics, Academic Press, Inc., 1979.

Junge, A., The telluric field in the Northern Germany induced by tidal motion in the North Sea, Geophysical Journal, 95, 523-533, 1988.

Larsen, J. C., Transport and heat flux of the Florida Current at $27 \mathrm{~N}$ derived from cross-stream voltages and profiling data: Theory and observations, Phil. Trans. R. Soc. Lond., A 338, 169-236, 1992.

Larsen, J. C., R. L. Mackie, A. Manzella, A. Fiodelisi, and S. Rieven, Robust smooth magnetotelluric transfer functions, Geophys. J. Int., 124, 801-819, 1996.

Longuet-Higgins, M. S., M. E. Stern, and H. Stommel, The electric field induced by ocean currents and waves, with applications to the method of towed electrodes, Papers in Physical Oceanography and Meteorology, XIII, No. 1, 1-37 (publ. by MIT and WHOI), 1954.

Palshin, N. A., L. L. Vanyan, and P. Kaikkonen, On-land amplification of the electric field induced by a coastal sea current, Phys. Earth Planet. Int., 94, 269-273, 1996.

Palshin, N. A., P. Kaikkonen, L. L. Vanyan, J. Tiikkainen, and V. H. Rukol, On-land detecting of a motionally induced electric field: test measurements in the Northern Finland, J. Geomag. Geoelectr., 49, 13431350, 1997.

Palshin, N. A., L. L. Vanyan, A. M. Poray-Koshits, Yu. V. Khan, P. Kaikkonen, J. Tiikkainen, V. A. Matyushenko, and L. R. Lukin, OnLand Measurements of the Motionally-Induced Electric Field, Oceanology, 39(3), 422-431, 1999.

Sanford, T. B., Motionally-induced electric and magnetic fields in the sea, J. Geophys. Res., 76, 3476-3492, 1971.

Vanyan, L. L., T. A. Demidova, I. V. Yegorov, and R. P. Bulatov, Numerical modeling of the electric field induced by Gulf Stream, Fizika Zemli, 4, 87-92, 1992 (in Russian).

Vanyan, L. L., H. Utada, H. Shimizu, Y. Tanaka, N. A. Palshin, V. Stepanov, V. Kouznetsov, R. D. Medzhitov, and A. A. Nozdrina, Studies on the lithosphere and the water transport by using the Japan Sea submarine cable (JASC): 1. Theoretical considerations, Earth Planets Space, 50, 35-42, 1998.

Viljanen, A. and L. Häkkinen, IMAGE magnetometer network, in SatelliteGround Based Coordination Sourcebook, edited by M. Lockwood, M. N. Wild, and H. J. Opgenoorth, pp. 111-117, ESA publications SP-1198, 1997.

Voit, S. S., V. V. Zhmur, and V. V. Fomin, Electromagnetic effects from along coast current of finite and infinite width, III Congress of Soviet oceanologists, Leningrad, Abstract book, pp. 41-42, 1987.

Yegorov, I. V. and N. A. Palshin, Numerical modeling of magnetotelluric fields in multilayer inhomogeneous medium, Phys. Solid Earth, 30(6), 540-545, 1994.

N. A. Palshin (e-mail: palshin@geo.sio.rssi.ru), L. L. Vanyan, A. M. Poray-Koshits, V. A. Matyushenko, P. Kaikkonen, and J. Tiikkainen 